Wright State University

CORE Scholar

1990

\title{
Transition Probabilities and Franck-Condon Factors for the Second Negative Band System of $\mathrm{O}_{2}^{+}$
}

Jane L. Fox

Wright State University - Main Campus, jane.fox@wright.edu

Alexander Dalgarno

Follow this and additional works at: https://corescholar.libraries.wright.edu/physics

Part of the Physics Commons

\section{Repository Citation}

Fox, J. L., \& Dalgarno, A. (1990). Transition Probabilities and Franck-Condon Factors for the Second Negative Band System of $\mathrm{O}_{2}{ }^{+}$. Journal of Geophysical Research: Space Physics, 95 (A2), 1119-1132. https://corescholar.libraries.wright.edu/physics/326

This Article is brought to you for free and open access by the Physics at CORE Scholar. It has been accepted for inclusion in Physics Faculty Publications by an authorized administrator of CORE Scholar. For more information, please contact library-corescholar@wright.edu. 


\title{
TRANSITION PROBABILITIES AND FRANCK-CONDON FACTORS FOR THE SECOND NEGATIVE BAND SYSTEM OF $\mathrm{O}_{2}^{+}$
}

\author{
J. L. Fox \\ Institute for Atmospheric Sciences and Department of Mechanical Engineering \\ State University of New York at Stony Brook
}

\section{A. Dalgarno}

Harvard-Smithsonian Center for Astrophysics, Cambridge

\begin{abstract}
Transition probabilities for the second negative band system of $\mathrm{O}_{2}^{+}$are computed using the dipole transition moment presented by Wetmore et al. [1984]. Vibrational levels $v^{\prime \prime}=0-54$ of the $X^{2} \Pi_{g}$ ground state and $v^{\prime}=0-33$ of the excited $A^{2} \mathrm{II}_{u}$ state are included. Franck-Condon factors for ionization-excitation of $\mathrm{O}_{2}$ $\left(X^{3} \Sigma_{g}^{-} ; v=0-25\right)$ to $\mathrm{O}_{2}^{+}\left(A^{2} \Pi_{u} ; v^{\prime}=0-33\right)$ are also presented.
\end{abstract}

\section{Introduction}

The second negative band system of $\mathrm{O}_{2}^{+}$arises from the transition $A^{2} \Pi_{u}\left(v^{\prime}\right) \rightarrow X^{2} \Pi_{g}\left(v^{\prime \prime}\right)$. The excited electronic state $A^{2} \Pi_{u}$ can be populated by photoionization and electron impact ionization of $\mathrm{O}_{2}$ and by fluorescent scattering of radiation by $\mathrm{O}_{2}^{+}\left(X^{2} \Pi_{g}\right)$ in planetary and cometary atmospheres. The latter process is a source of excitation of high vibrational levels $(v>5-7)$ of ground state $\mathrm{O}_{2}^{+}$ in the atmospheres of the terrestrial planets [Fox, 1985, 1986]. Recently, James et al. [1988] and Schappe et al. [1988] have investigated the production of the second negative band system by electron impact on $\mathrm{O}_{2}$. They have presented cross sections for production of specific bands within the system and for the system as a whole. Analysis of the emission spectrum requires Franck-Condon factors for the excitation-ionization of ground state $\mathrm{O}_{2}$ to produce $\mathrm{O}_{2}^{+}\left(A^{2} \Pi_{u}\right)$ and radiative transition probabilities for individual bands of the second negative system. Since transition probabilities were not available, both James et al. and Schappe et al. used Franck-Condon factors to compute the ratios of band intensities, a practice which is valid only if the transition moment is constant with internuclear distance. In addition, in computing the FranckCondon factors, James et al. used a Morse potential, which is less accurate for high vibrational levels than a Rydberg-Klein-Rees (RKR) potential.

Several years ago, in connection with studies of the vibrational distribution of $\mathrm{O}_{2}^{+}$in the dayside ionospheres of the terrestrial planets [Fox, 1985; 1986], we computed transition probabilities of the second negative band sys-

Copyright 1990 by the American Geophysical Union.

Paper number 89JA01115.

0148-0227/90/89JA-01115\$02.00 tem, using the ab initio dipole transition moment of Wetmore et al. [1984]. Wetmore et al. [1984] presented absorption oscillator strengths for the bands involving the first eight vibrational levels of the ground and excited states, and lifetimes of the $\mathrm{O}_{2}^{+}\left(A^{2} \Pi_{u}\right)$ state for $v^{\prime}=$ $0-33$. The computed lifetimes agreed very well with the available measured values [Jeunehomme, 1966; Erman and Larsson, 1977; Fink and Welge, 1968]. We present here transition probabilities for vibrational levels $v^{\prime \prime}=0$ to 54 of the ground state $\mathrm{O}_{2}^{+}\left(X^{2} \Pi_{g}\right)$ and $v^{\prime}=0$ to 33 of $\mathrm{O}_{2}^{+}\left(A^{2} \Pi_{\mathrm{u}}\right)$. We also present Franck-Condon factors for ionization-excitation of $\mathrm{O}_{2}\left(X^{3} \Sigma_{g}^{-} ; v=0-\right.$ 25) to produce $\mathrm{O}_{2}^{+}\left(A^{2} \Pi_{u} ; v^{\prime}=0-33\right)$, a wider range of vibrational quantum numbers than previously available [Nicholls, 1965; Krupenie, 1972]. Many vibrational quanta are potentially important, because the equilibrium internuclear distance of the $\mathrm{O}_{2}^{+}\left(A^{2} \Pi_{u}\right)$ potential curve is very different from those of the $\mathrm{O}_{2}\left(X^{3} \Sigma_{g}^{-}\right)$and $\mathrm{O}_{2}^{+}\left(X^{2} \Pi_{g}\right)$ states. Hence a wide range of vibrational levels of the $A$ state are populated in ionization or fluorescent scattering; the excited states then decay to an even wider range of vibrational levels of the ground state.

\section{Calculations and Results}

We have constructed vibrational wave functions for the $\mathrm{O}_{2}^{+}\left(X^{2} \Pi_{g}\right), \mathrm{O}_{2}^{+}\left(A^{2} \Pi_{u}\right)$ and $\mathrm{O}_{2}\left(X^{3} \Sigma_{g}^{-}\right)$states by numerical integration of the radial equation of nuclear motion [cf. Wetmore et al. 1984]. Our potential curves for $\mathrm{O}_{2}^{+}$ were adopted from the RKR values of Krupenie [1972] extended to larger internuclear distances using the asymptotic polarization term $-\frac{1}{2} \alpha R^{-4}$, where $\alpha=5.28 a_{0}^{3}$ is the polarizability of atomic oxygen. The potential curves for the ground state of $\mathrm{O}_{2}$ were taken from Allison et al. [1982]. The Franck-Condon factors for ionizationexcitation of $\mathrm{O}_{2}\left(X^{3} \Sigma_{g}^{-} ; v=0-25\right)$ to $\mathrm{O}_{2}^{+}\left(A^{2} \Pi_{u} ; v^{\prime \prime}=\right.$ $0-33$ ) are presented in Tables $1 a-1 c$. Our values for ionization-excitation from $\mathrm{O}_{2}\left(X^{3} \Sigma_{g}^{-} ; v=0\right)$ agree well with those presented by James et al. [1988].

Transition probabilities and band origins of the second negative system are given in Tables $2 a-2 e$ for $\mathrm{O}_{2}^{+}\left(A^{2} \mathrm{II}_{\mathrm{u}}\right.$, $\left.v^{\prime}=0-33\right)$ and $\mathrm{O}_{2}^{+}\left(X^{2} \Pi_{g}, v^{\prime \prime}=0-54\right)$. Vibrational levels of the $X$ state with $v^{\prime \prime} \geq 30$ lie above the lowest vibrational level of the $A$ state, so some of the high vibrational levels of the $X$ state decay radiatively to vibrational levels of the $A$ state. The "reverse" $X \rightarrow A$ 


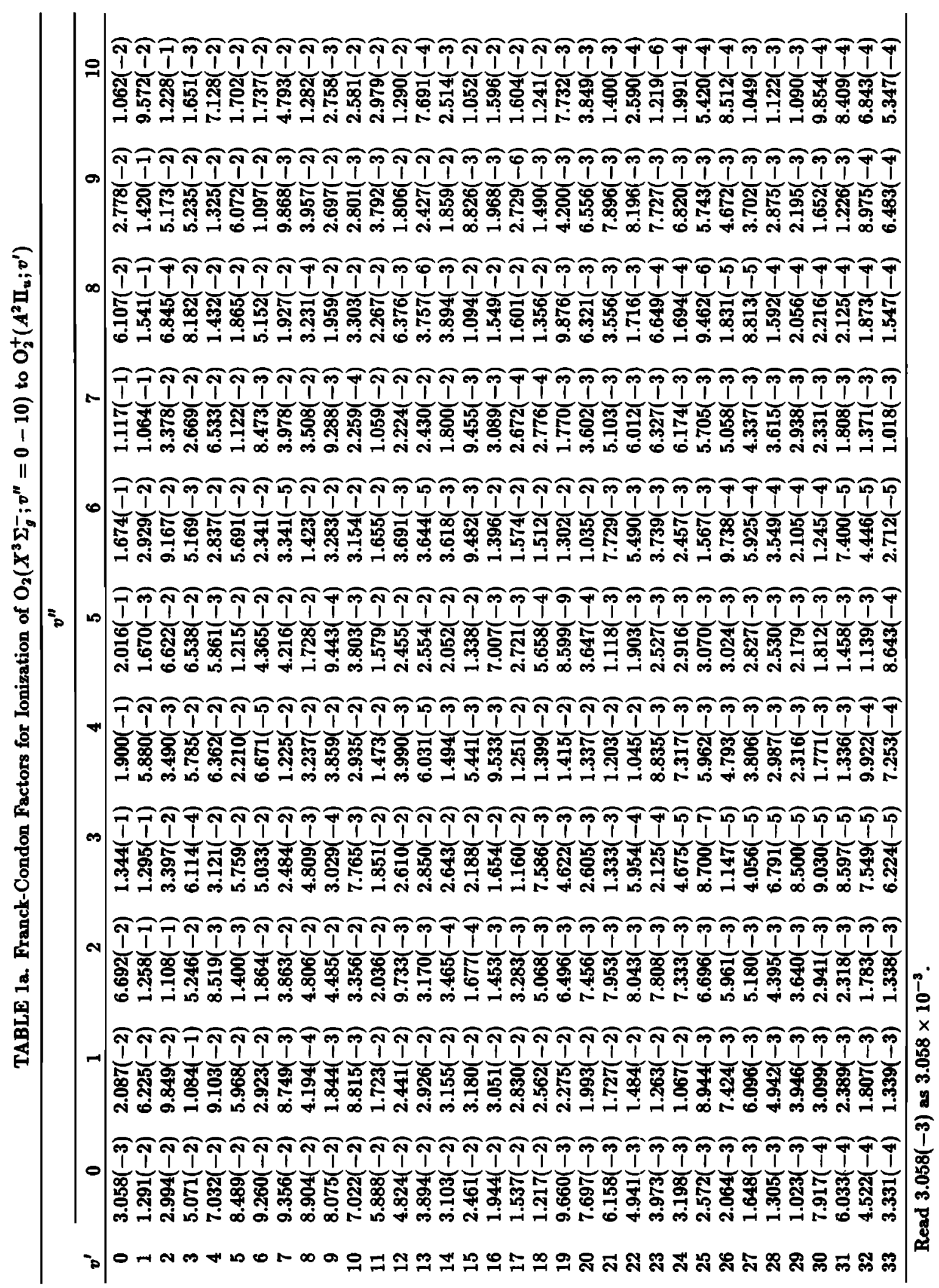




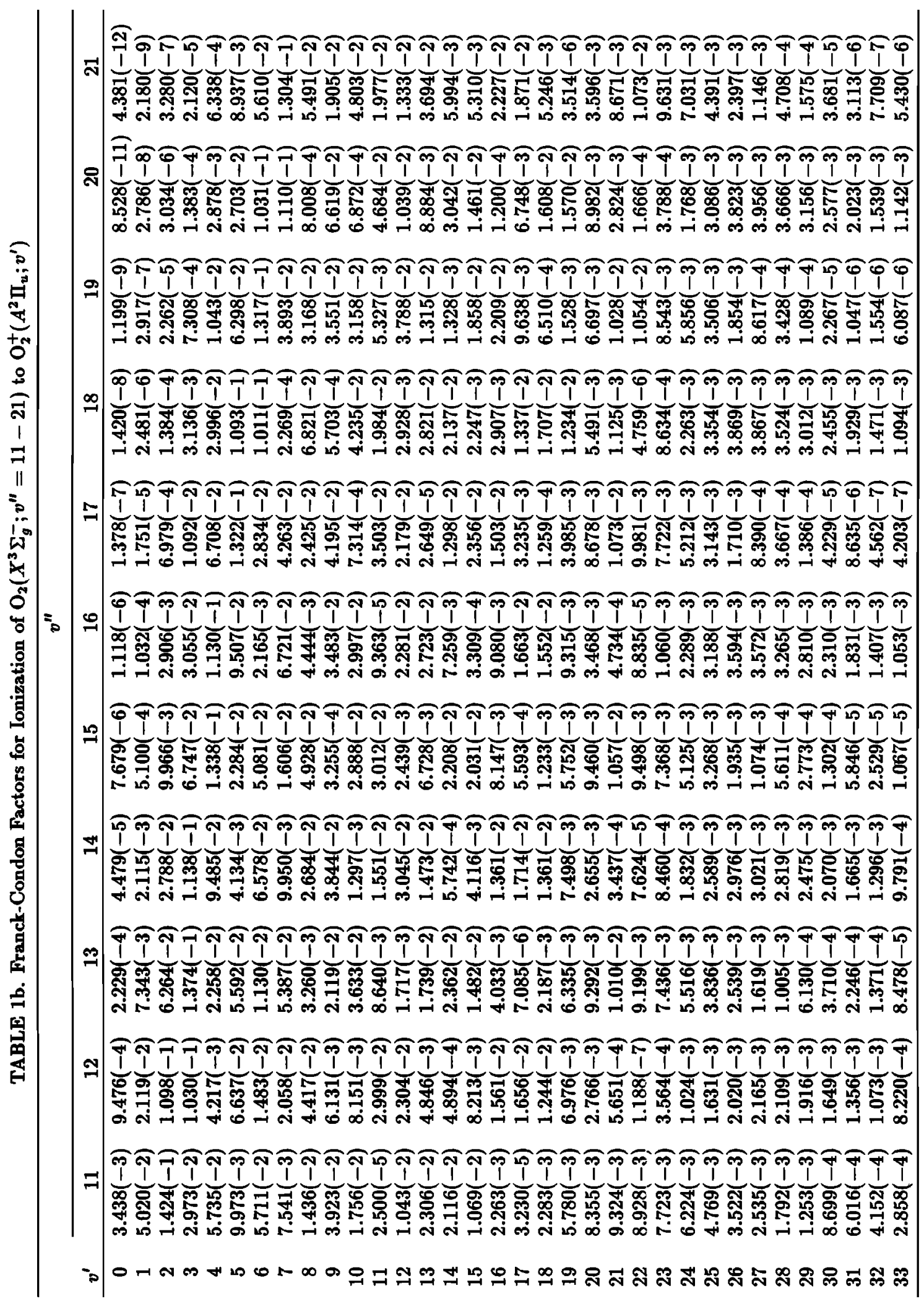


TABLE 1c. Franck-Condon Factors for Ionization of $\mathrm{O}_{2}\left(X^{3} \Sigma_{g}^{-} ; v^{\prime \prime}=22-25\right)$ to $\mathrm{O}_{2}^{+}\left(A^{2} \Pi_{u} ; v^{\prime}\right)$

\begin{tabular}{rcccc}
\hline & \multicolumn{4}{c}{$v^{\prime \prime}$} \\
$v^{\prime}$ & 22 & 23 & 24 & 25 \\
\hline 0 & $1.734(-13)$ & $1.402(-14)$ & $1.358(-15)$ & $1.458(-15)$ \\
1 & $1.301(-10)$ & $5.255(-12)$ & $3.902(-13)$ & $2.301(-14)$ \\
2 & $2.842(-8)$ & $1.915(-9)$ & $8.125(-11)$ & $5.250(-12)$ \\
3 & $2.597(-6)$ & $2.497(-7)$ & $1.865(-8)$ & $9.033(-10)$ \\
4 & $1.114(-4)$ & $1.547(-5)$ & $1.660(-6)$ & $1.365(-7)$ \\
5 & $2.308(-3)$ & $4.675(-4)$ & $7.378(-5)$ & $8.873(-6)$ \\
6 & $2.258(-2)$ & $6.909(-3)$ & $1.624(-3)$ & $2.926(-4)$ \\
7 & $9.406(-2)$ & $4.720(-2)$ & $1.737(-2)$ & $4.786(-3)$ \\
8 & $1.224(-1)$ & $1.266(-1)$ & $8.221(-2)$ & $3.720(-2)$ \\
9 & $6.715(-3)$ & $7.602(-2)$ & $1.330(-1)$ & $1.193(-1)$ \\
10 & $5.900(-2)$ & $7.356(-3)$ & $2.053(-2)$ & $1.016(-1)$ \\
11 & $6.192(-3)$ & $5.850(-2)$ & $4.635(-2)$ & $4.604(-4)$ \\
12 & $4.703(-2)$ & $9.229(-3)$ & $1.657(-2)$ & $6.410(-2)$ \\
13 & $3.429(-3)$ & $2.321(-2)$ & $4.265(-2)$ & $1.985(-3)$ \\
14 & $1.634(-2)$ & $3.251(-2)$ & $1.707(-4)$ & $3.272(-2)$ \\
15 & $2.951(-2)$ & $1.487(-3)$ & $2.373(-2)$ & $2.542(-2)$ \\
16 & $8.420(-3)$ & $1.061(-2)$ & $2.612(-2)$ & $1.064(-7)$ \\
17 & $4.815(-4)$ & $2.378(-2)$ & $3.776(-3)$ & $1.602(-2)$ \\
18 & $1.057(-2)$ & $1.456(-2)$ & $2.530(-3)$ & $2.333(-2)$ \\
19 & $1.708(-2)$ & $2.260(-3)$ & $1.369(-2)$ & $1.046(-2)$ \\
20 & $1.343(-2)$ & $6.014(-4)$ & $1.683(-2)$ & $6.189(-4)$ \\
21 & $6.132(-3)$ & $5.578(-3)$ & $1.097(-2)$ & $1.808(-3)$ \\
22 & $1.245(-3)$ & $9.781(-3)$ & $4.001(-3)$ & $7.144(-3)$ \\
23 & $6.771(-6)$ & $1.044(-2)$ & $4.421(-4)$ & $1.021(-2)$ \\
24 & $9.526(-4)$ & $8.500(-3)$ & $1.820(-4)$ & $9.795(-3)$ \\
25 & $2.404(-3)$ & $5.760(-3)$ & $1.414(-3)$ & $7.443(-3)$ \\
26 & $3.440(-3)$ & $3.381(-3)$ & $2.696(-3)$ & $4.808(-3)$ \\
27 & $3.830(-3)$ & $1.747(-3)$ & $3.422(-3)$ & $2.736(-3)$ \\
28 & $3.697(-3)$ & $7.957(-4)$ & $3.551(-3)$ & $1.396(-3)$ \\
29 & $3.259(-3)$ & $3.133(-4)$ & $3.269(-3)$ & $6.430(-4)$ \\
30 & $2.698(-3)$ & $1.015(-4)$ & $2.780(-3)$ & $2.668(-4)$ \\
31 & $2.134(-3)$ & $2.358(-5)$ & $2.238(-3)$ & $9.849(-5)$ \\
32 & $1.631(-3)$ & $2.278(-6)$ & $1.731(-3)$ & $3.133(-5)$ \\
33 & $1.213(-3)$ & $2.267(-7)$ & $1.296(-3)$ & $7.972(-6)$ \\
\hline & & & & \\
\cline { 2 - 4 } & & &
\end{tabular}

transitions are indicated by negative band origins and transition probabilities. For strong bands with fairly low vibrational quantum number, intensity ratios computed with our transition probabilities agree to within a few percent with those computed with the Franck-Condon factors of James et al. [1988] and Schappe et al. [1988]. Significant differences arise for weaker bands and higher vibrational levels.

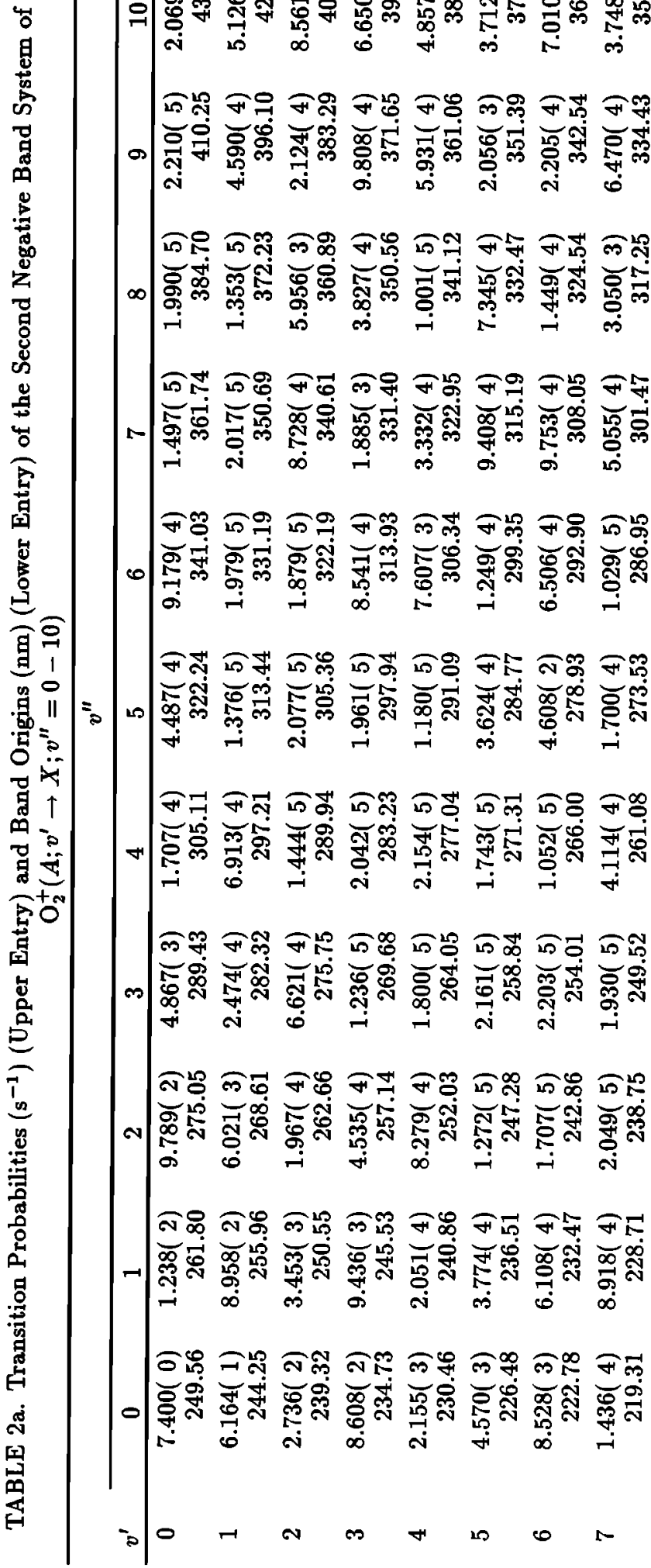

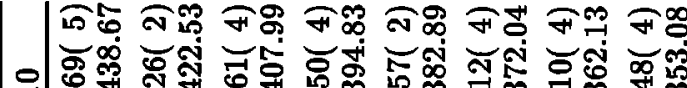

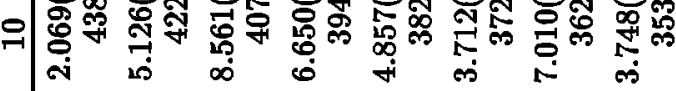

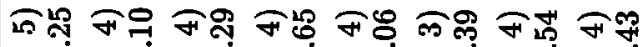

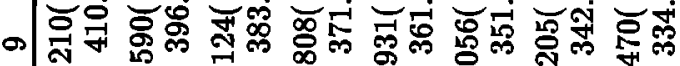

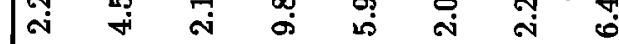

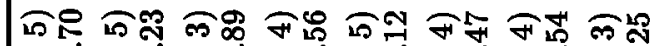

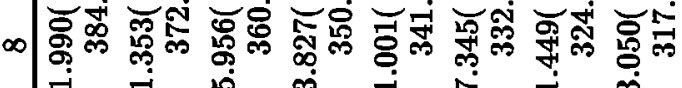

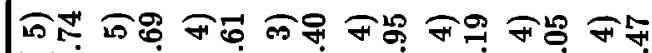

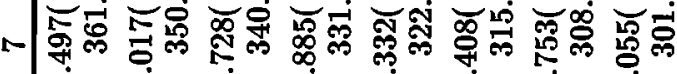

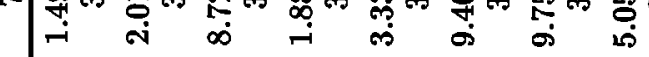

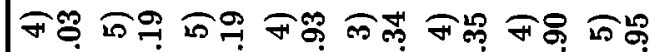

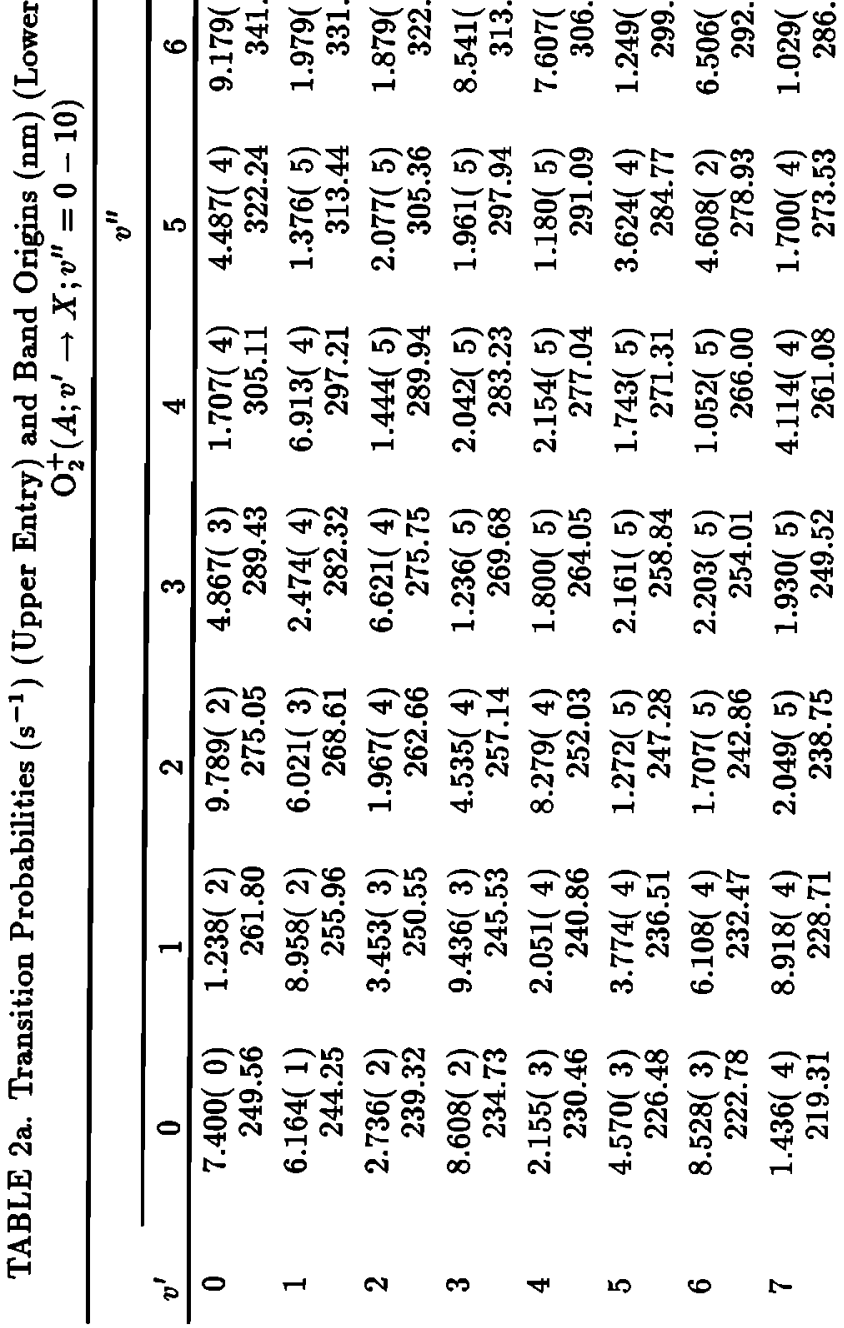




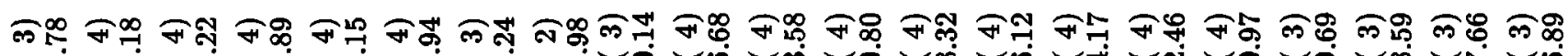

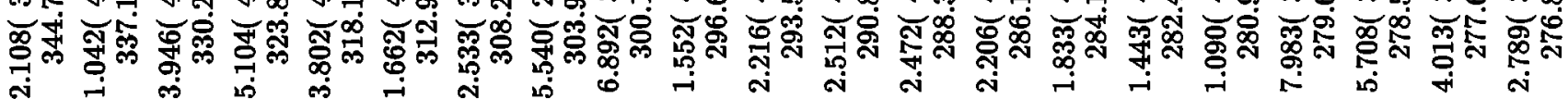

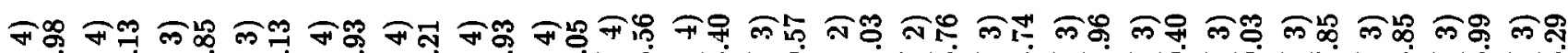

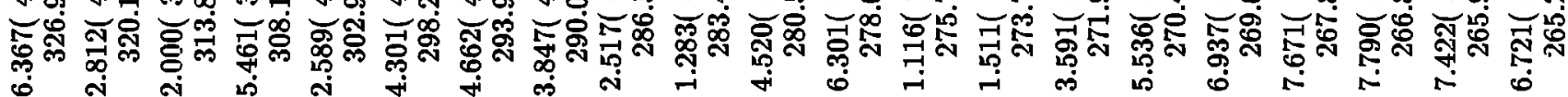

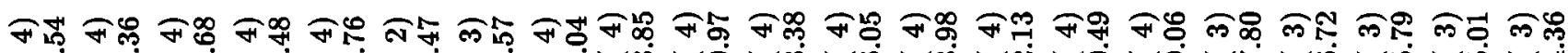

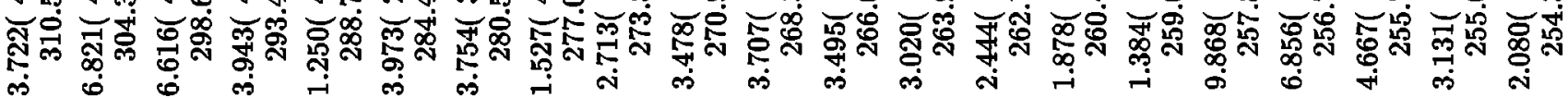

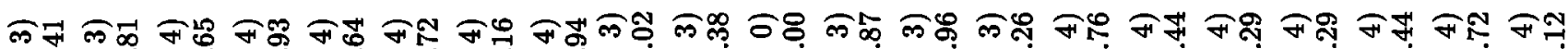

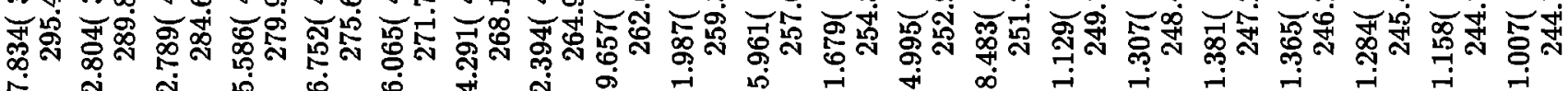

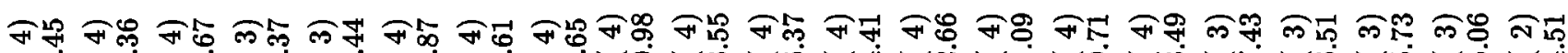

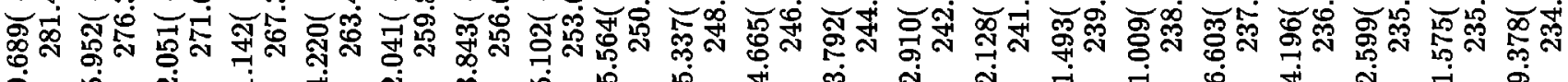

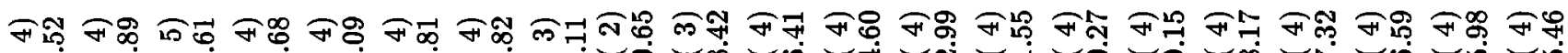

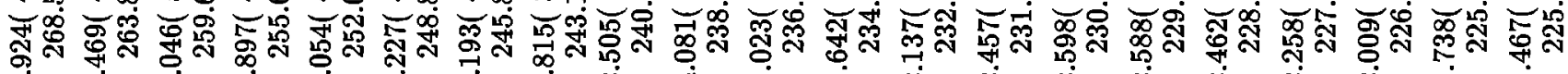
สํำ

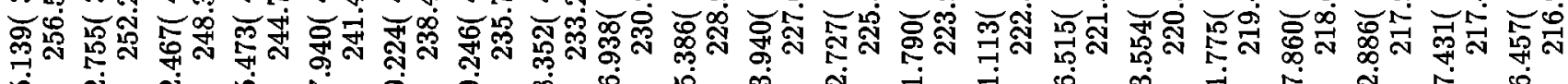

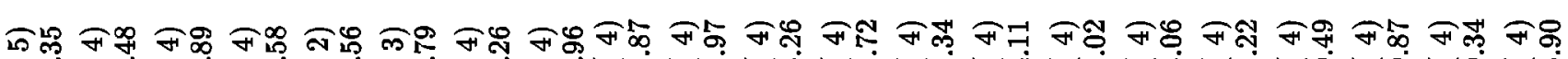

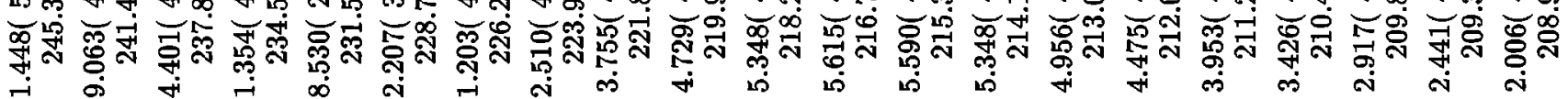

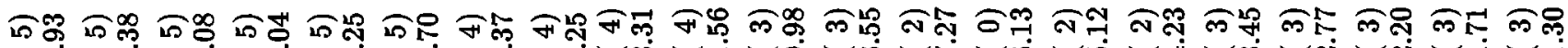

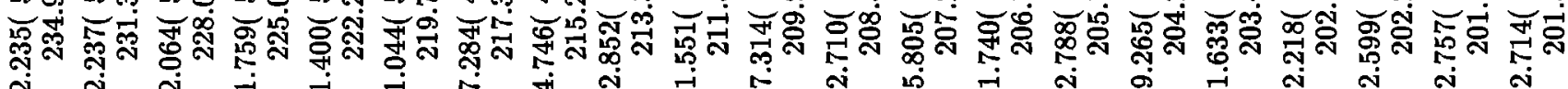

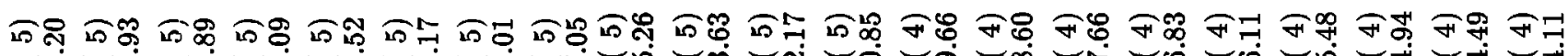
庐

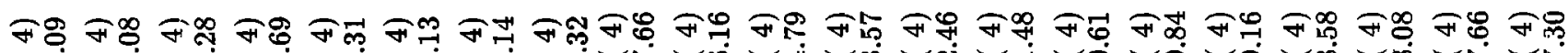

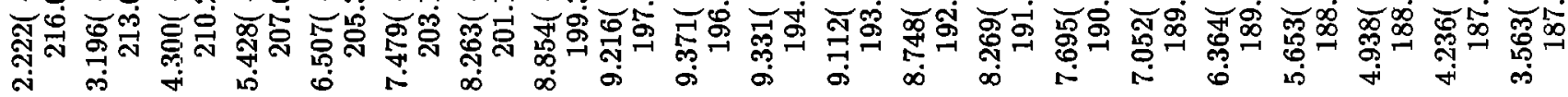

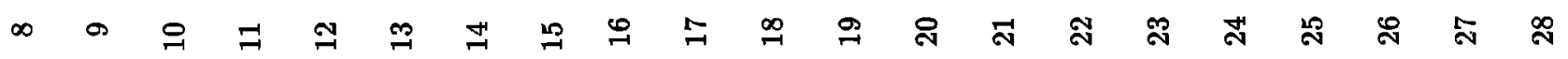




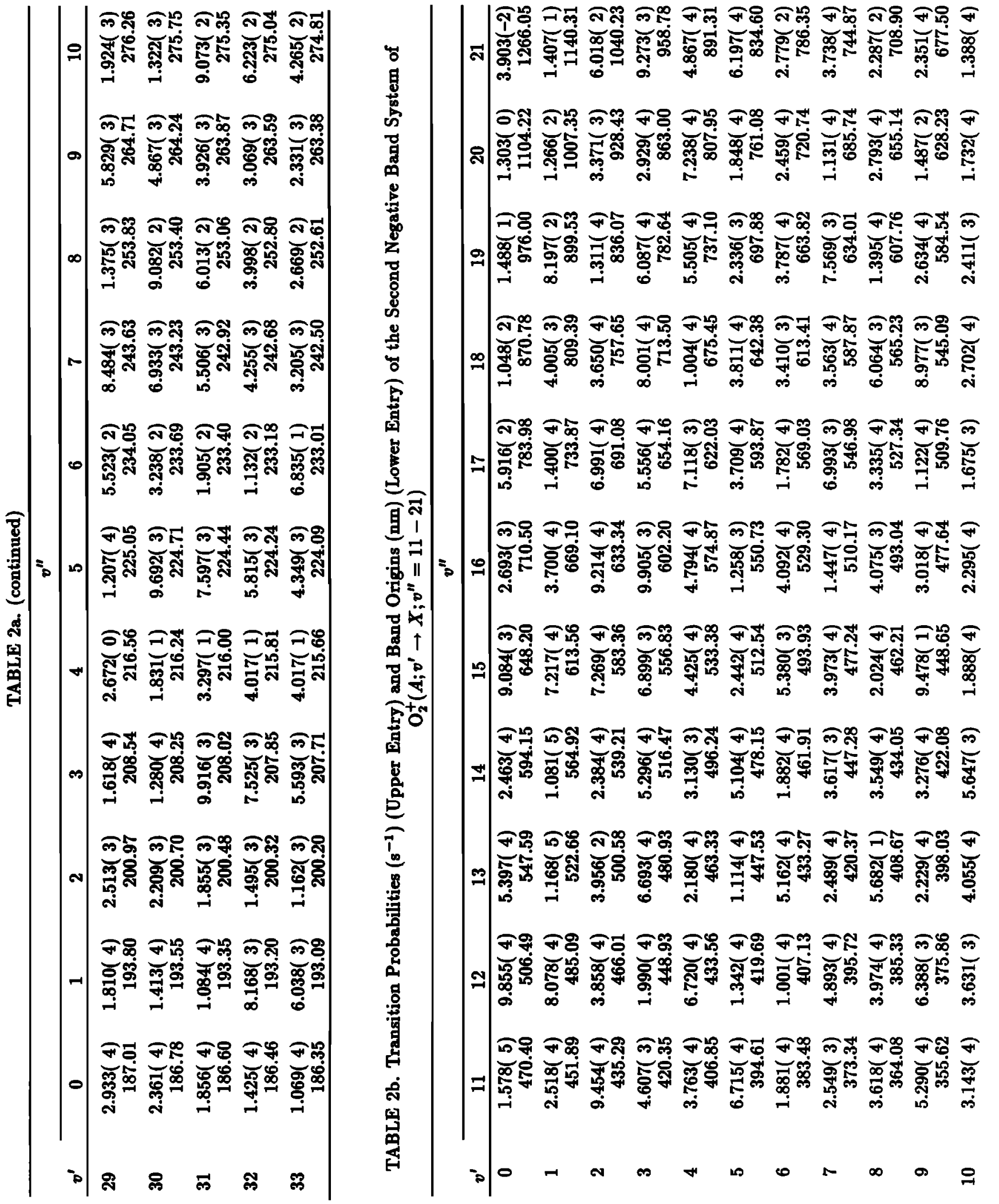




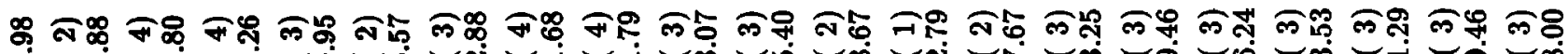
产 不

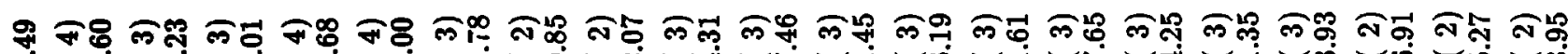

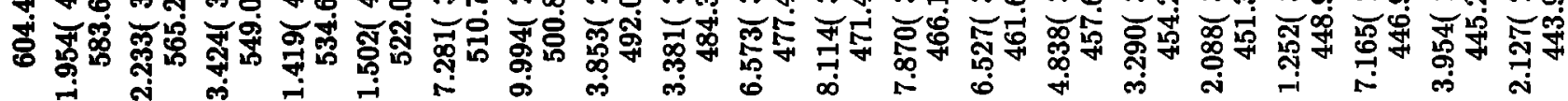

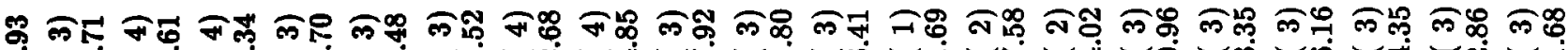

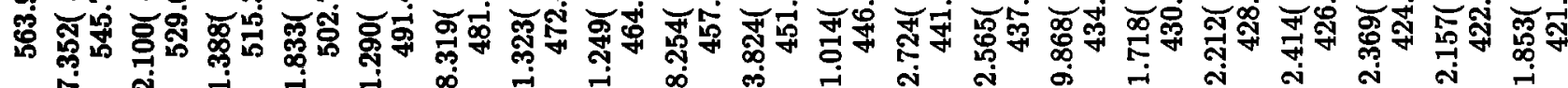

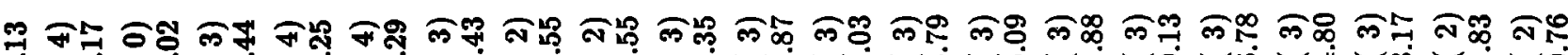

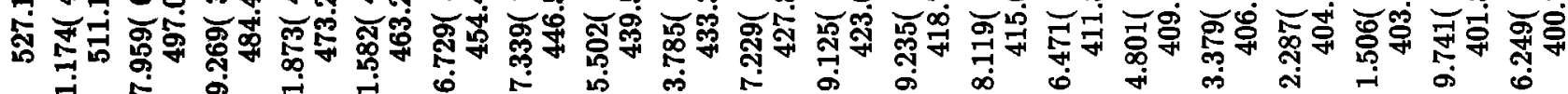

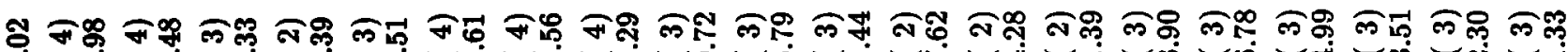

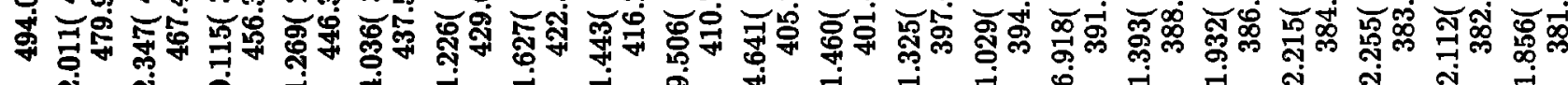

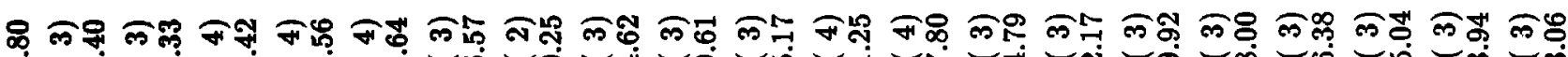

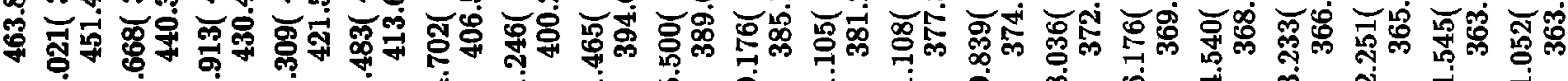

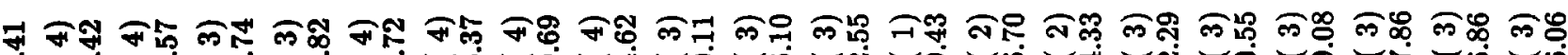

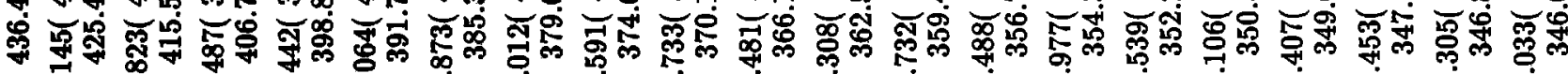

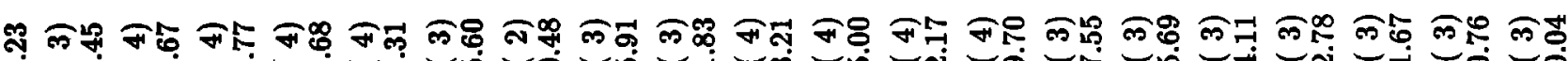

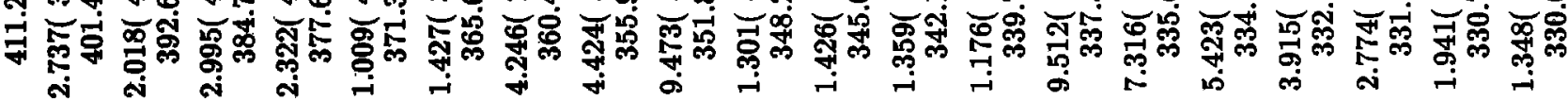

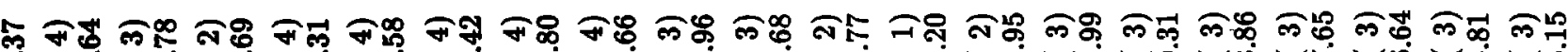

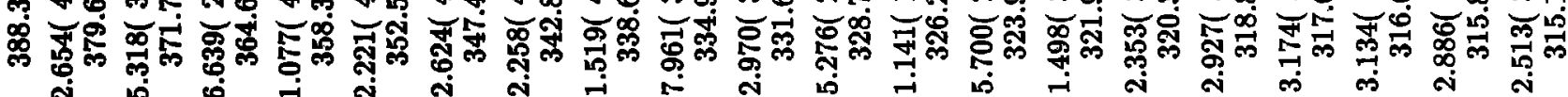

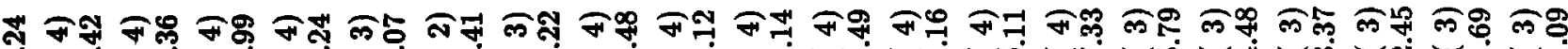

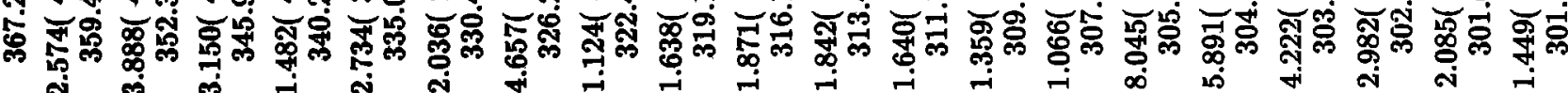

品

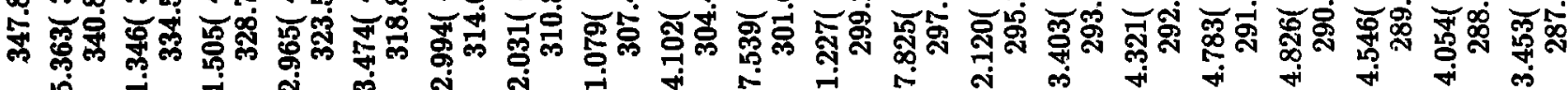

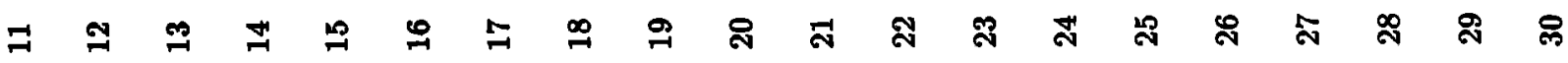




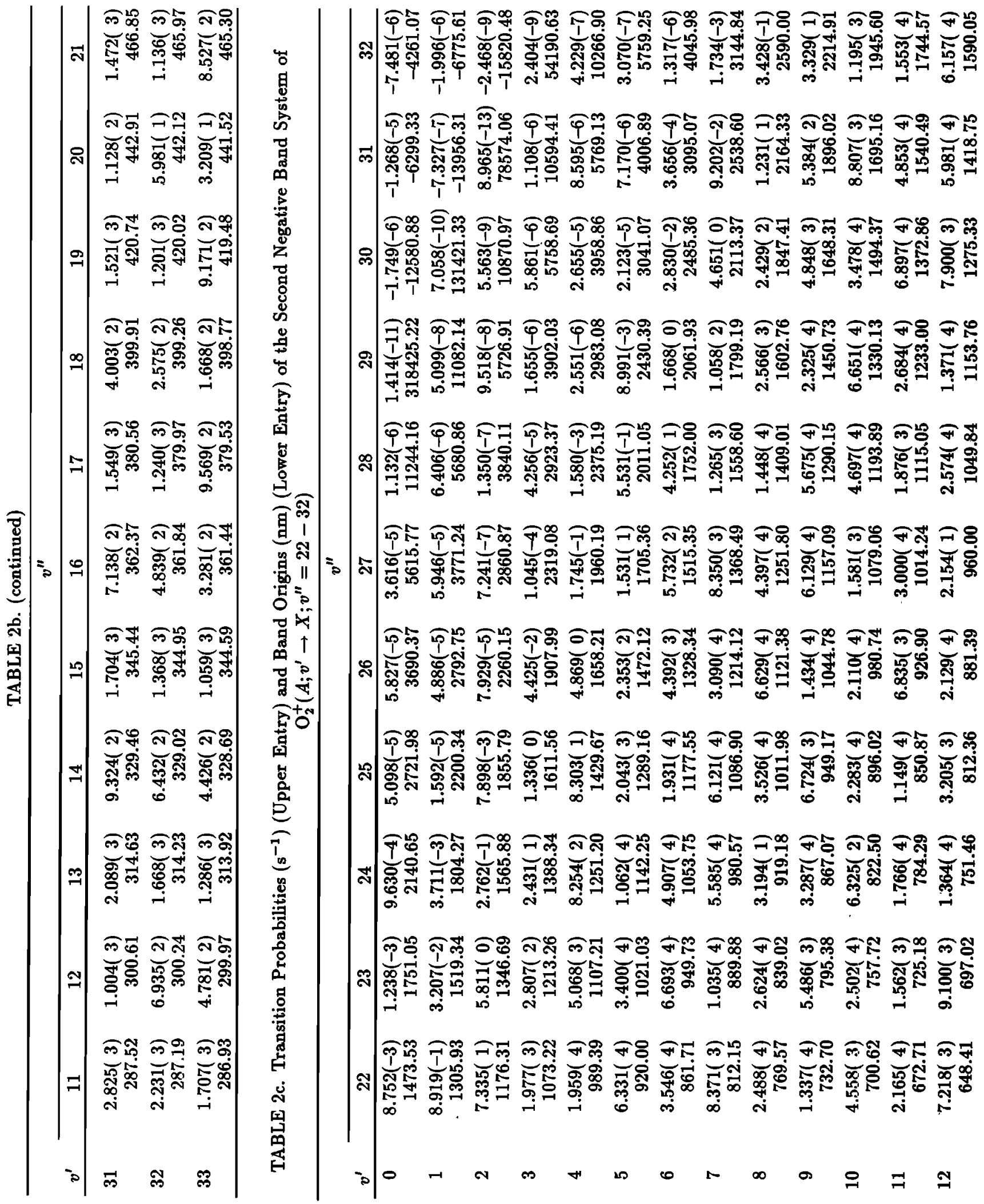




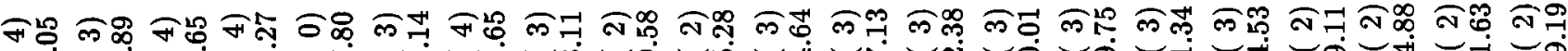
鹿嵒 施

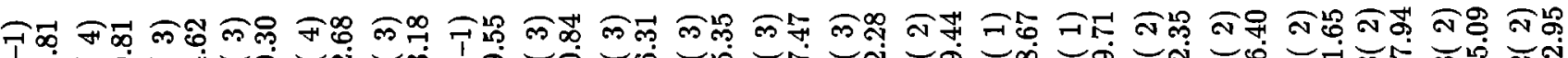

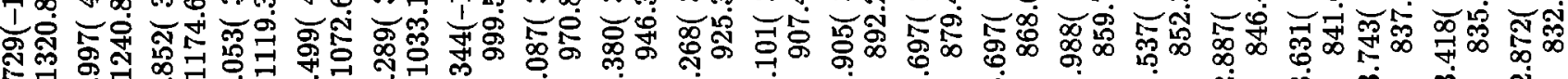

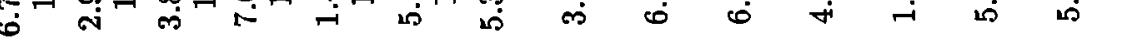

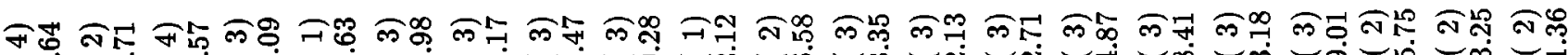

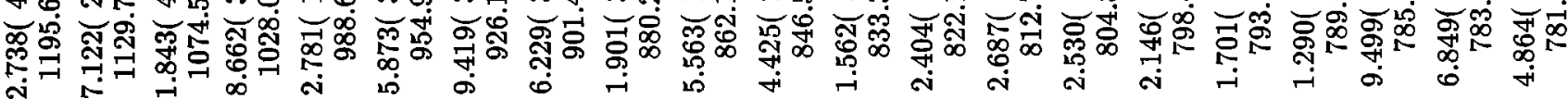

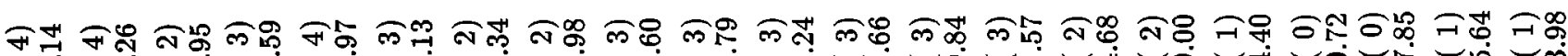

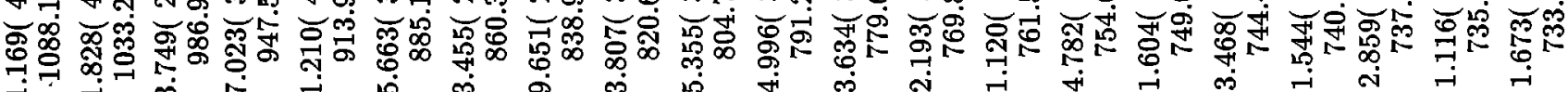
ต๓

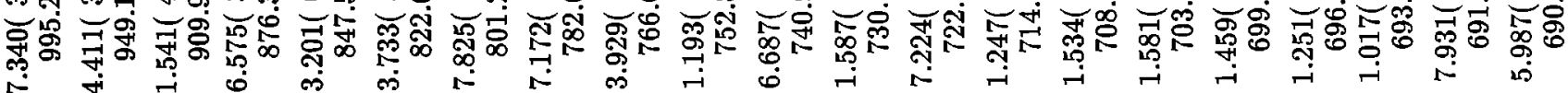

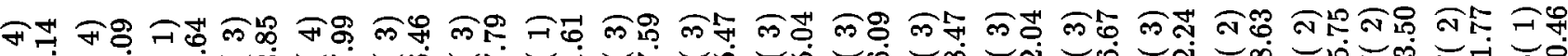

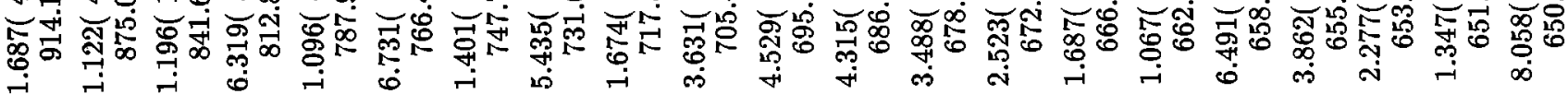

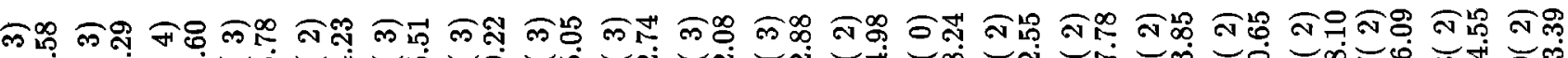

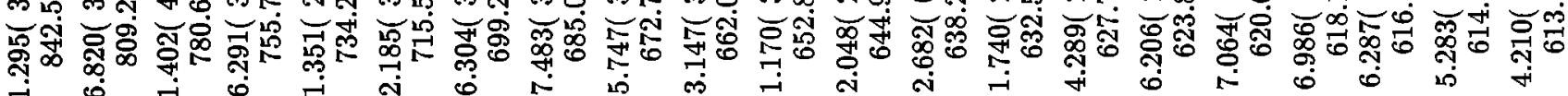

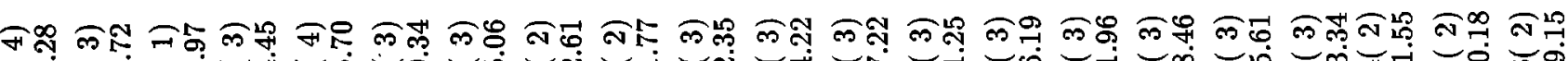

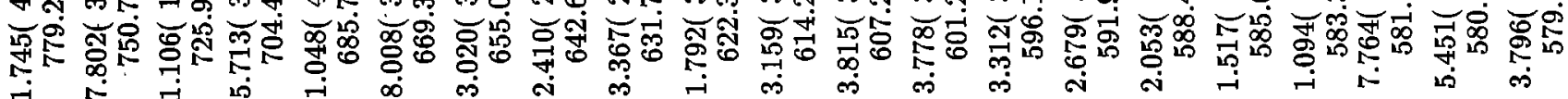

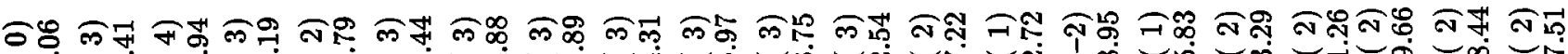

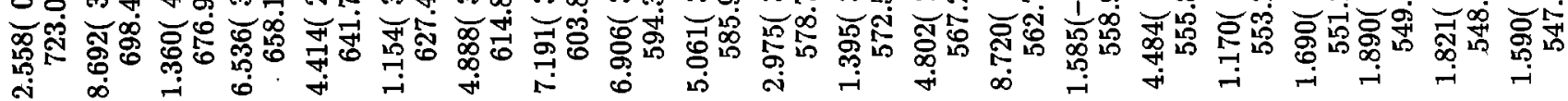

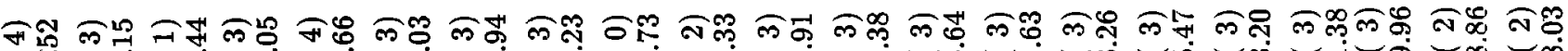

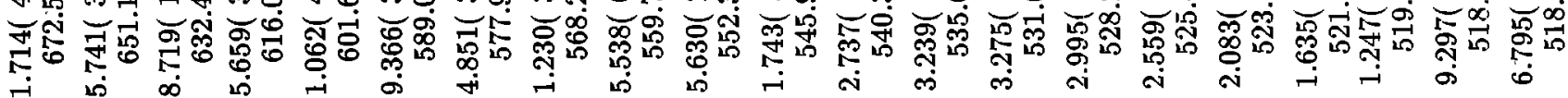

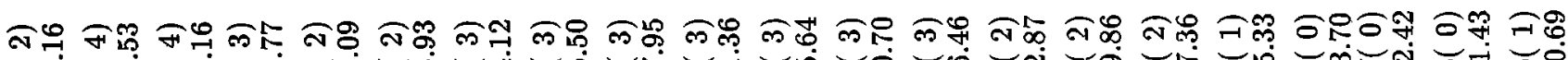
顿

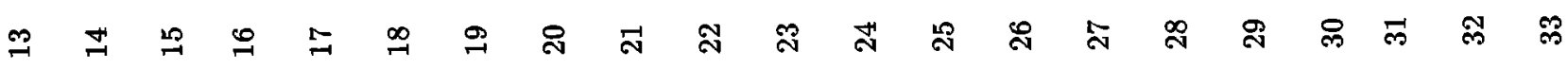




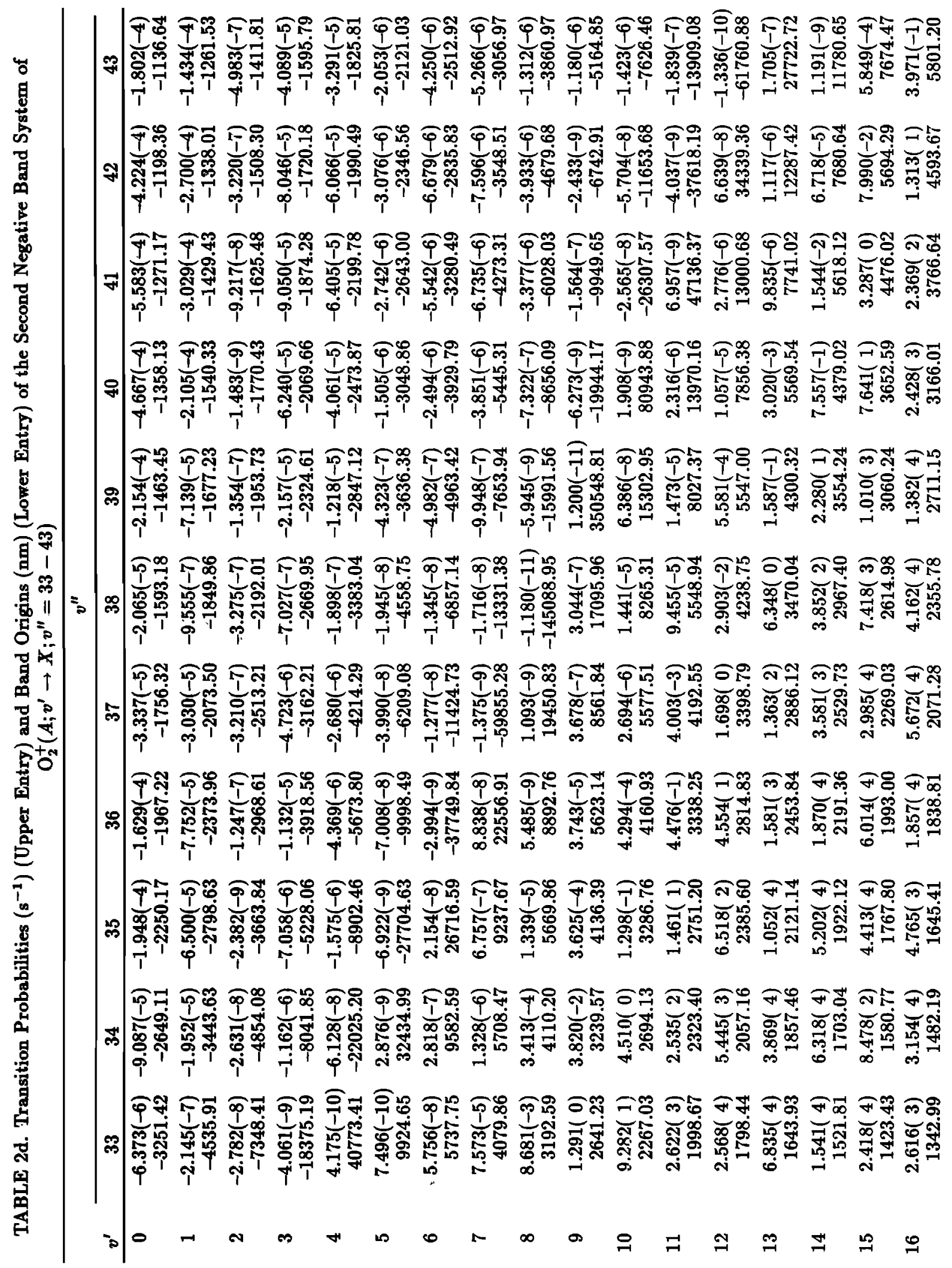


규

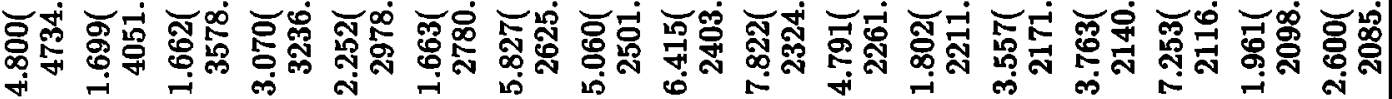

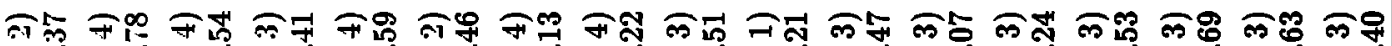

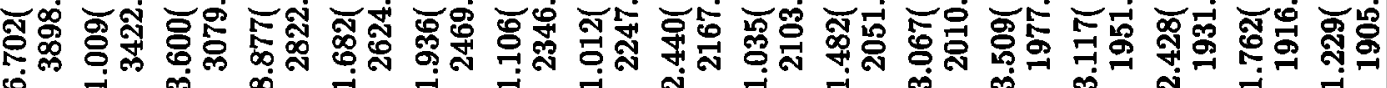

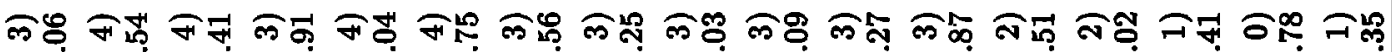

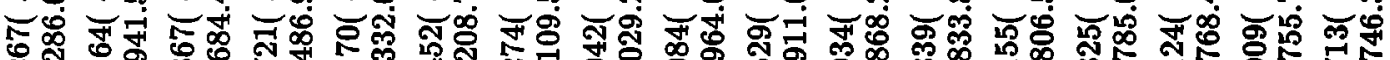

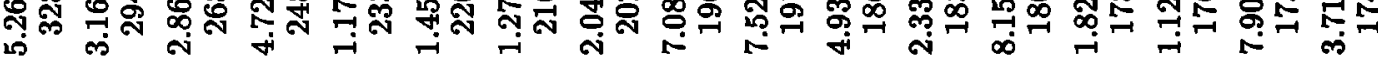

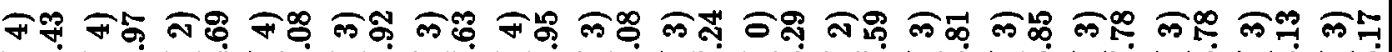

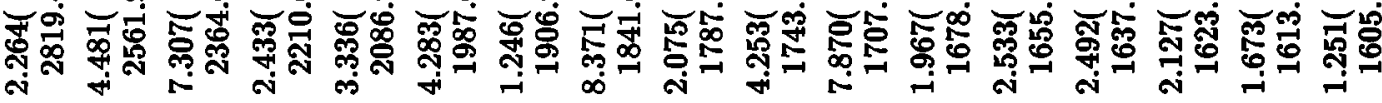

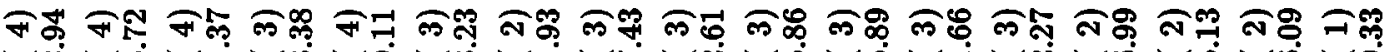

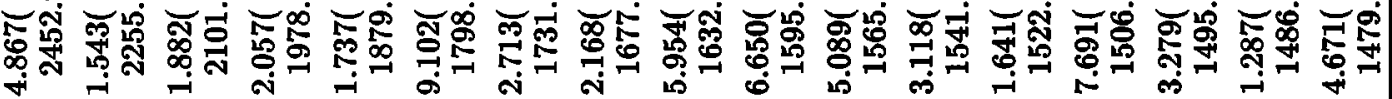

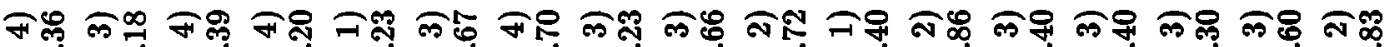

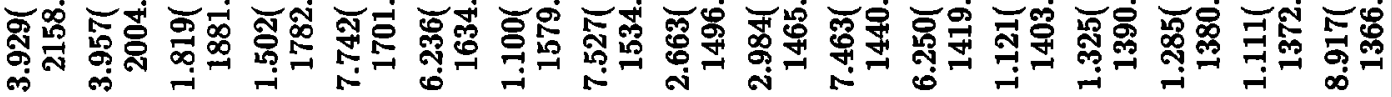

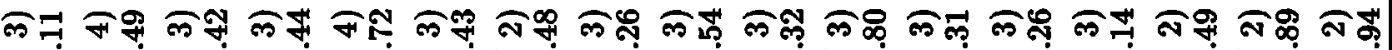

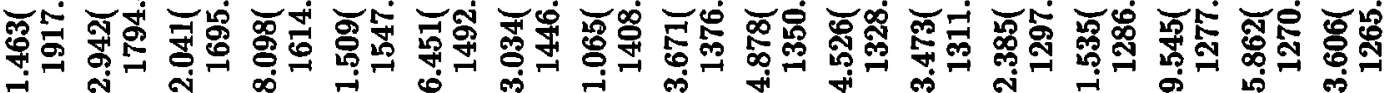

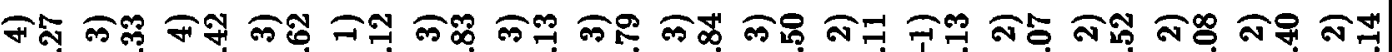

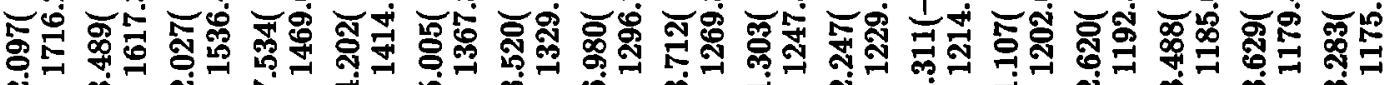

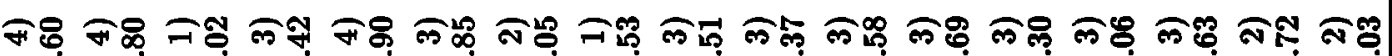

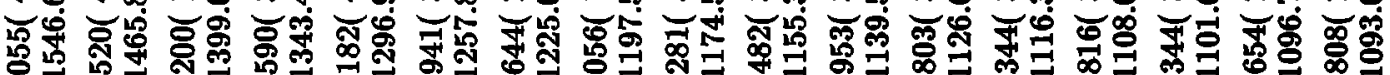

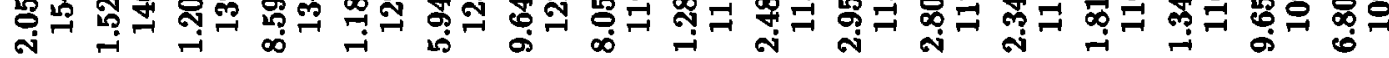

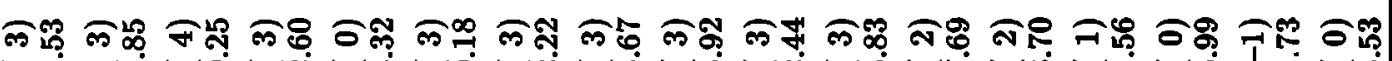

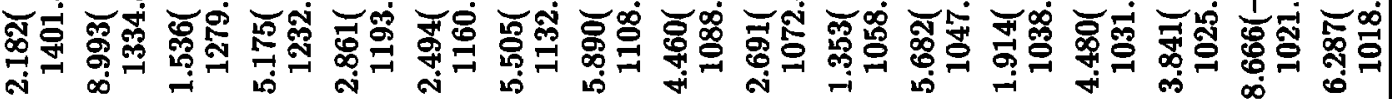

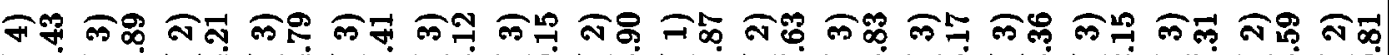

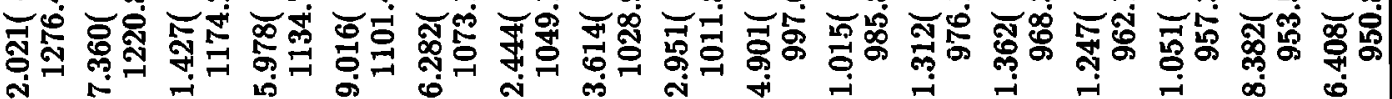

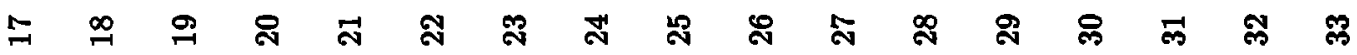




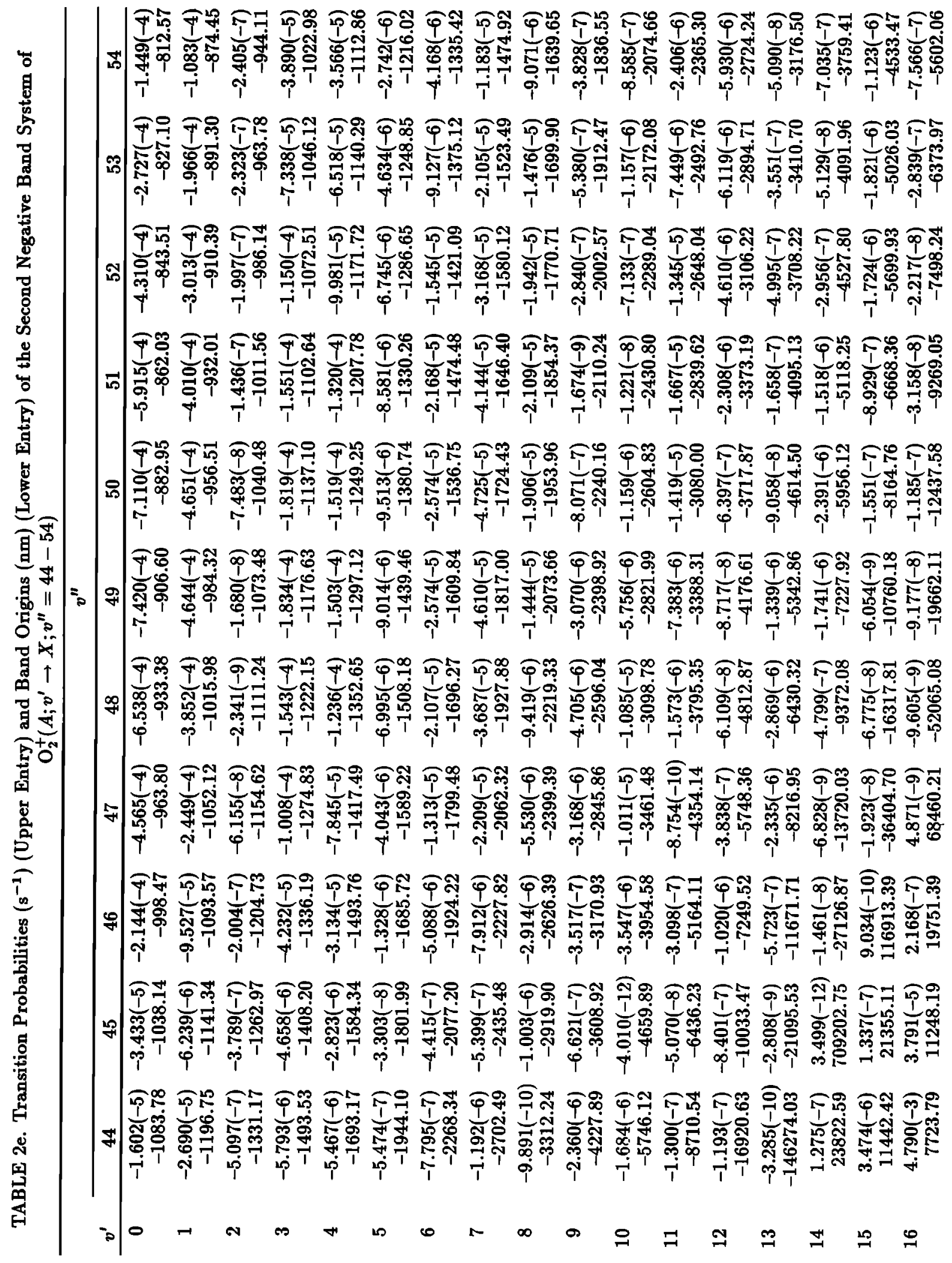




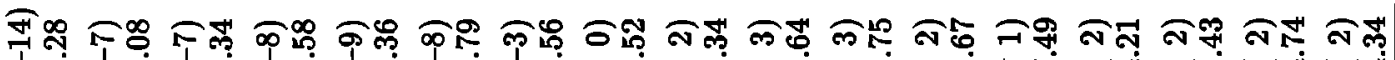

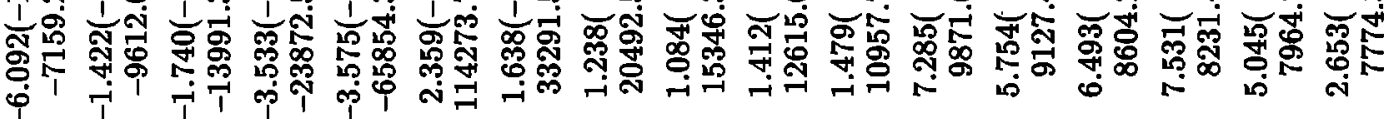

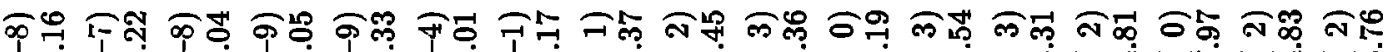

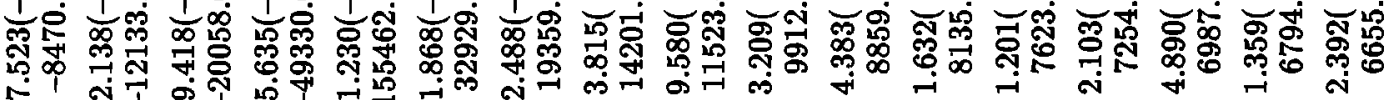

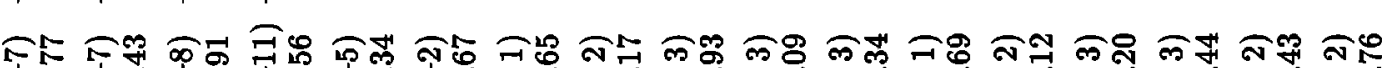

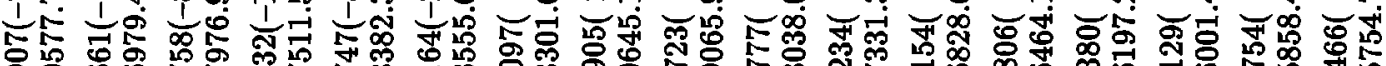

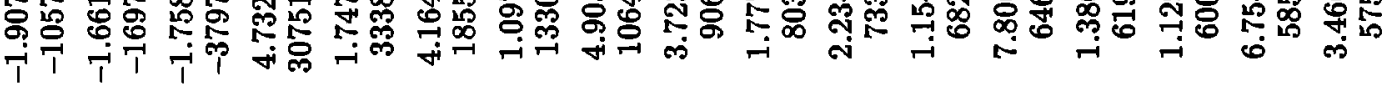
ㄷำ

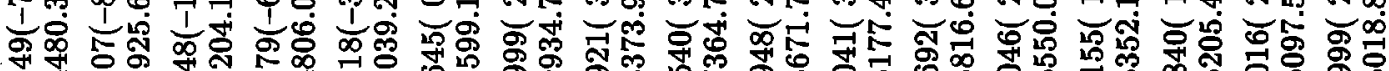

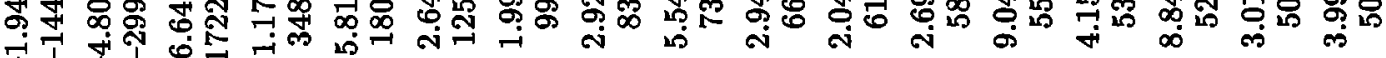
11 个

कㅇ 유

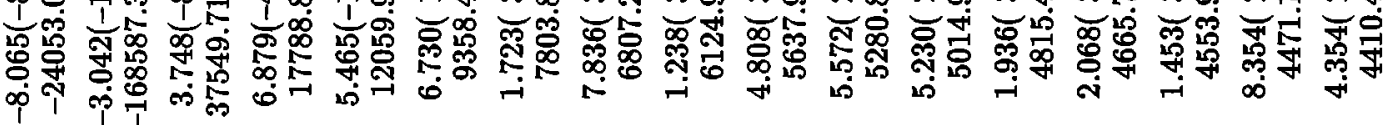

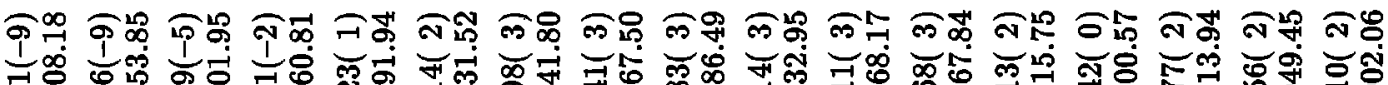

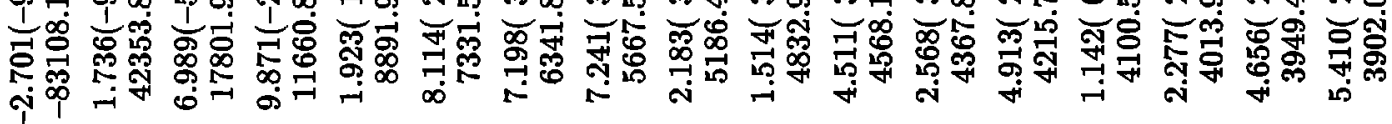

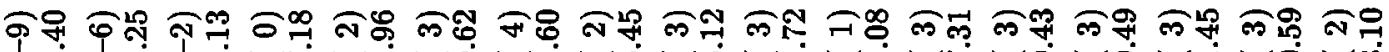

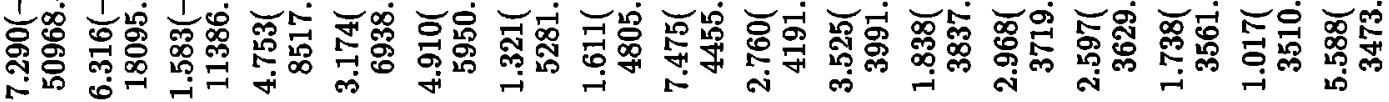

金踝

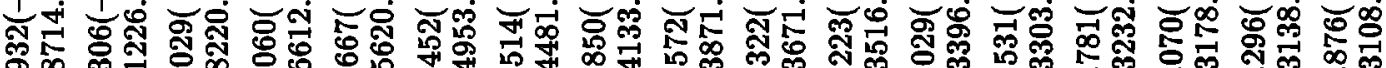

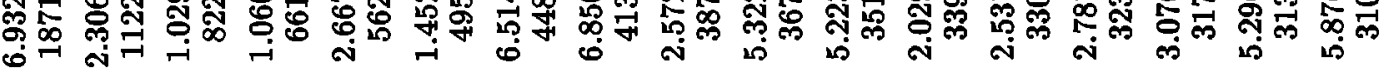

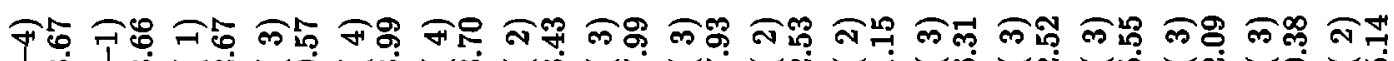
잉

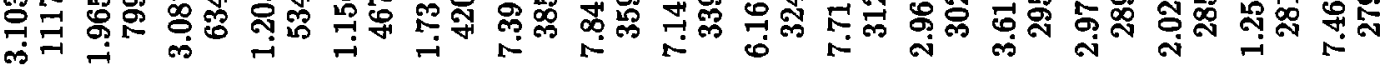

สิธ

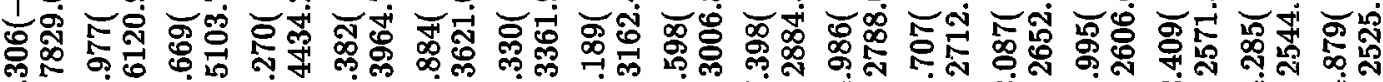

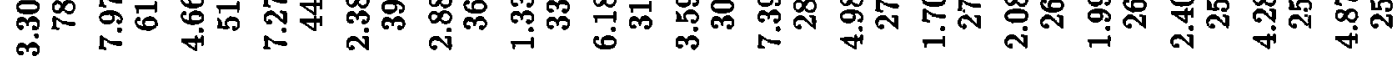

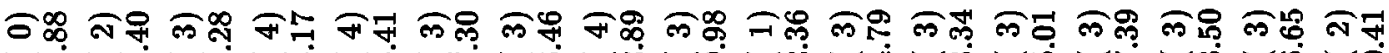

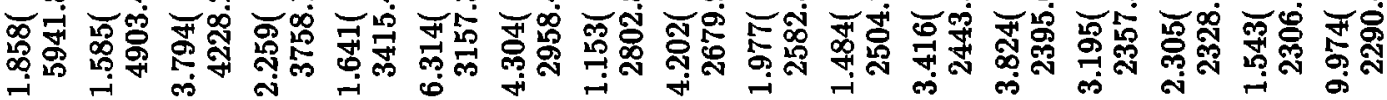

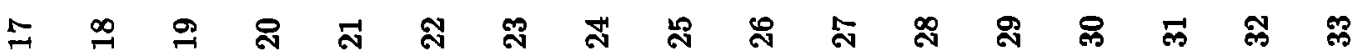


Acknowledgments. This work was supported in part by the National Science Foundation under grant ATM8700436 and by the National Aeronautics and Space Administration under grants NAGW-665 and NAGW-1561.

The Editor thanks two referees for their assistance in evaluating this paper.

\section{References}

Allison, A. C., S. L. Guberman, and A. Dalgarno, Photoabsorption into the ${ }^{3} \Pi_{u}$ state of $\mathrm{O}_{2}, J$. Geophys. Res., 87, 923, 1982.

Erman, P., and M. Larsson, Lifetimes of some excited levels in some important ion-molecules, part II, $\mathrm{O}_{2}^{+}$, Phys. Scr., 15, 335, 1977.

Fink, E. H., and K. H. Welge, Lebensdauern und Löschquerschnitte elektronisch angeregter Zustände von $\mathrm{N}_{2} \mathrm{O}, \mathrm{NO}, \mathrm{O}_{2}^{+}, \mathrm{CO}^{+}$and $\mathrm{CO}, Z$. Naturforsch $A$., 23, 358, 1968.

Fox, J. L., The $\mathrm{O}_{2}^{+}$vibrational distribution in the Venusian ionosphere, Adv. Space Res., 5, 165, 1985.

Fox, J. L., The vibrational distribution of $\mathrm{O}_{2}^{+}$in the dayside ionosphere, Planet. Space Sci., I \&, 1241, 1986.

James, G. K., J. M. Ajello, D. E. Shemansky, B. Franklin, D. Siskind, and T. G. Slanger, An investigation of the second negative system of $\mathrm{O}_{2}^{+}$by electron impact, $J$. Geophys. Res., 93, 9893, 1988.

Jeunehomme, M., Oscillator strengths of the negative systems of oxygen, J. Chem. Phys., 44, 4253, 1966.

Krupenie, P. H., The spectrum of molecular oxygen, $J$. Phys. Chem. Ref. Data, 1, 423, 1972.

Nicholls, R. W., Franck-Condon factors for the $\mathrm{O}_{2}^{+}$first and second negative band systems, Can. J. Phys., 49, 1390, 1965 .

Schappe, R. S., M. B. Schulman, F. A. Sharpton, and C. C. Lin, Emission of the $\mathrm{O}_{2}^{+}\left(A^{2} \Pi_{u} \rightarrow X^{2} \Pi_{g}\right)$ secondnegative-band system produced by electron impact on $\mathrm{O}_{2}$, Phys. Rev., 38, 4537, 1988.

Wetmore, R. W., J. L. Fox and A. Dalgarno, Radiative lifetimes of the second negative system of $\mathrm{O}_{2}^{+}$, Planet. Space Sci., 92, 1111, 1984.

A. Dalgarno, Harvard-Smithsonian Center for Astrophysics, Cambridge, MA 02138

J. L. Fox, Department of Mechanical Engineering, State University of New York at Stony Brook, Stony Brook, NY 11794.

(Received February 27, 1989;

revised May 30, 1989;

accepted June 1, 1989.) 